\title{
A Corpus-Based Study on English Synonyms Convention and Tradition
}

\author{
Yuan-Fan LI \\ School of Foreign Language, Wuhan Textile University, Wuhan 430070, China
}

fannylyflyf@163.com

Keywords: Corpus, Collocation, Semantic Prosody, Tradition, Convention.

\begin{abstract}
For English learners, the discrimination of synonyms is a big problem, but corpus is an effective tool to clear the difference between them. It offers English learners an authentic, simple and straightforward way to overcome the difficulties within synonyms. In this paper, the researcher uses a corpus-based method to study the difference between two English words --- tradition and convention, by using the qualitative and quantitative analysis to analyze the collocation and semantic prosody of these two words. According to the analysis of the researcher, tradition is more frequently used in subjects related to religion and culture and more widely used in written English, especially in literary or academic works while convention is more frequently used in subjects related to politics and social life and more widely used in spoken English, but they all hold neutral semantic prosody. A corpus-based study is helpful to English teaching and learning.
\end{abstract}

\section{Introduction}

In modern linguistics, corpus (pl. corpora) refers to a collection of texts or parts of texts upon which some general linguistic analysis can be conducted [1]. Due to the widespread access of the technology, corpus linguistics has become a prominent part in modern linguistics. Corpus linguistics is not a separate discipline of linguistics but a linguistic methodology and a specific scientific study of linguistic phenomena. It uses large electronic databases of 'real life' language to examine hypotheses about language use and linguistics theories. Within corpus linguistics, there are various views as to the value of corpus annotation, from John Sinclair[2], advocating minimal annotation and allowing texts to speak for themselves, to others, such as Wallis and Nelson advocating annotation as a path to greater linguistic understanding[3]. Corpus linguistics believes that "words did not have inherent meanings, but depended on their environments to select or at least confirm their meaning" [4]. The corpus approach then looks for collocational meaning in vocabulary. This approach is particularly helpful for the teaching and learning of synonyms.

Synonyms refer to different words with almost identical or similar meanings. Some lexicographers claim that no synonyms have exactly the same meaning. Corpus-based studies show that near synonyms may share similar meanings but they have different collocation behavior [5]. For example, powerful and strong share similar meaning, but as to collocations, people usually use powerful car and strong tea instead of powerful tea and strong car [6]. Moreover, the semantic prosody of near synonyms may not be the same. For example, fickle and flexible carries positive and negative prosody, respectively [7].

Researches have been done to study the characteristics of actual use of synonyms of Chinese EFL learners. Corpus-based studies show that near-synonyms differ in their collocational behavior and semantic prosody which causes deviation in Chinese EFL learners' English using [8]. However, most of the researches focus on the theoretical overview of corpus application. Few empirical studies are found on corpus approach to the teaching and learning of near synonyms. Accordingly, an empirical study should be carried out to investigate the effectiveness of the corpus-based approach on the teaching and learning of near-synonyms.

In this paper, the researcher uses a corpus-based method to study the difference between two English words --- tradition and convention, by using the qualitative and quantitative analysis to analyze the collocation and semantic prosody of these two words. 


\section{Frequency and Collocation}

As to the frequency of tradition and convention in different registers, the author classified them into Spoken, Fiction, Magazine, Newpaper and Academic according to the data provided by Corpus of Comtemporary American English (COCA). The frequency of this pair of near-synonyms in the five registers is shown in Table 1.

Table 1. Frequency of tradition and convention in COCA

\begin{tabular}{lrrrrr}
\hline & Spoken & Fiction & Magazine & Newspaper & Academic \\
\hline \multirow{2}{*}{ tradition } & {$[2735]$} & {$[1419]$} & {$[6149]$} & {$[4399]$} & {$[10918]$} \\
& & & & & \\
convention & {$[5841]$} & {$[670]$} & {$[2832]$} & {$[4246]$} & {$[4770]$} \\
\hline
\end{tabular}

By searching high-frequency collocations of tradition and convention, the author finds that the high-frequency collocations of tradition are long, American, Christian, oral, western, great, catholic, religious, old and new while those of convention are national, democratic, center, republican, Geneva, visitors, bureau, Baptist, annual and international.

The statistical data of the frequency of tradition and convention in different registers and the search of their high-frequency collocations leads to the conclusion that tradition is more frequently used in subjects related to religion and culture and more widely used in written English, especially in literary or academic works. However, convention is more frequently used in subjects related to politics and social life and more widely used in spoken English. It reveals that there is a correlation between the occurrence frequency of synonyms and the tendency of language system, also fully explains the existence of register priority of synonyms in actual use.

\section{Semantic Prosody}

Semantic prosody describes the way in which certain seemingly neutral words can be perceived with positive or negative associations through frequent occurrences with particular collocations. It can be classified into positive prosody, neural prosody and negative prosody[9]. If a keyword occurs more frequently with negative words or expressions, this keyword has negative prosody. If a keyword occurs more frequently with positive words or expressions, it has positive prosody. If it occuts with both, it has neural prosody.

The high-frequency occurrences of tradition are long, American, Christian, oral, western, catholic, religious, old and new. These words don't have positive or negative prosody. In addition, the author randomly selects the concordance of tradition in COCA and lists them below.

Yet the tradition does reflect the communitarian vision that is necessary for...

According to ancient tradition, it was the titan Prometheus who, having stolen fire from the...

How important is it to you to see that tradition maintained?

It's a well-worn tradition observed by generations of cotton farmers in Montezuma and...

Poor Brazilians have a long tradition of coming up with derisive tags for rich kids.

New Hampshire primary day finally arrived, the tiny hamlet of Dixville Notch cast its tradition first votes at midnight.

These great singers in the $\mathrm{R} \& \mathrm{~B} /$ soul tradition.

Yes, I mean, this is something of a Syrian tradition.

We're in the tradition of cautionary tales.

We are going to lay our daughter to rest in the confines and the tradition of what we do...

The above concordance shows that the occurrences of tradition have no specific semantic preference. Therefore, tradition has neural prosody. 
The high-frequency occurrences of convention are national, democratic, center, republican, Geneva, visitors, bureau, Baptist, annual and international. These words don't have positive or negative prosody. The author also randomly selects the concordance of convention in COCA and lists them below.

In Phoenix, where Gore spoke to an advertising industry convention about Current TV.

Then instead of bidding the patient good day, as convention dictates, he helps her cope with the experience of...

The last thing you want somebody doing is going off message in primetime at a convention.

To win some of Nevada's national delegates when party leaders abruptly shut down the convention.

They got a very good review on a convention which was -- this is not original, but it was ...

IP uses this convention to resolve the hierarchical structure of IP addresses and experience has shown it allows for

The new convention -- like a classical column consisting of a base, a shaft, and a...

But Poe and Wyatt obeyed the usual convention and began with the most "primitive" -- hence the reversed...

Poster presented at the annual convention of the American Speech-Language-Hearing Association, Philadelphia, PA

Portions of this work were presented at the 2009 American Speech-Language-Hearing Association Convention in New Orleans, Louisiana.

The above concordance shows that the occurrences of tradition have no specific semantic preference. Therefore, tradition has neural prosody.

\section{Conclusion}

This paper analyses the near-synonyms tradition and convention based on data from COCA. The statistical data of the frequency of tradition and convention in different registers and the search of their high-frequency collocations leads to the conclusion that tradition is more frequently used in subjects related to religion and culture and more widely used in written English, especially in literary or academic works while convention is more frequently used in subjects related to politics and social life and more widely used in spoken English. It also shows that there is a correlation between the occurrence frequency of synonyms and the tendency of language system, and the existence of register priority of synonyms in actual use. The analysis of the concordances of this pair of near-synonyms reveals that they both have neural prosody.

\section{References}

[1] C. F. Meyer, English Corpus Linguistics: An Introduction, Cambridge University Press, Cambridge, 2002.

[2] J. Sinclair, The automatic analysis of corpora, in: J. Svartvik (Ed.), Directions in Corpus Linguistics (Proceedings of Nobel Symposium 82), Mouton de Gruyter, Berlin, 1992.

[3] S. Wallis and G. Nelson, Knowledge discovery in grammatically analysed corpora, Data Mining and Knowledge Discovery. 5 (2001) 307-340.

[4] G. Francis and J.McH. Sinclair, I bet he drinks Carling Black Label: A riposte to Owen on corpus grammar, Applied Linguistics. 15 (1994) 190-200.

[5] J. Conzett, Integrating collocation into a reading and writing course, in: J. Coady and T. Huckins (Eds.), Second Language Vocabulary Application, CUP, Cambridge, 1997, pp. 70-87.

[6] M. A. K. Halliday, System and Function in Language, OUP, Oxford, 1976.

[7] E. Tognini-Bonelli, Corpus Linguistics at Work, John Benjamins, Amsterdam, 2001. 
[8] Lu Jun, A corpus-based study on collocational vehavior and semantic prosody of near synonyms in Chinese learner English, Modern Foreign Languages. 33 (2010)276-286.

[9] M. Stubbs, Text and Corpus Analysis, Blackwell Publishers Ltd., Oxford, 1996. 